\title{
Acute pulmonary edema due to hypoxia during a difficult intubation in a rheumatoid arthritis patient
}

\author{
Ji Seon Jeong, Jong Hun Jun, Hyo Jin Song, and Sung Hwan Choi \\ Department of Anesthesiology and Pain Medicine, Hanyang University School of Medicine, Seoul, Korea
}

We experienced a rheumatoid arthritis (RA) patient who developed acute pulmonary edema by hypoxia in the absence of spontaneous respiratory effort by sufficient muscle relaxation during anesthesia induction.

A 61-year-old, $136 \mathrm{~cm}, 48 \mathrm{~kg}$ female patient was treated at our hospital for a bilateral total knee arthroplasty due to RA involving the knee joints. During preoperative evaluation of the patient, her chest radiograph showed a suspicious bronchitis at right lower lobe costophrenic angle area (Fig. 1A), whereas the other laboratory tests showed no specific abnormality.

The patient was moved to the operating room to receive bilateral total knee replacement. After normal tidal breathing of $100 \%$ oxygen $6 \mathrm{~L} / \mathrm{min}$ for 3 min using a tightly fitting face mask, anesthesia was induced by injecting $60 \mathrm{mg}$ of propofol and 0.3 $\mu \mathrm{g} / \mathrm{kg} / \mathrm{min}$ of remifentanil and $30 \mathrm{mg}$ of rocuronium. Under mask ventilation, intubation was performed after $3 \mathrm{~min}$. Intubation was performed using Fiberoptic intubation due to difficulties with endotracheal intubation based on the preoperative cspine radiography finding of atlantoaxial subluxation. Fiberoptic intubation was tried for $3 \mathrm{~min}$ but failed, the subsequent mask ventilation was performed for $3 \mathrm{~min}$, during which the patient's ventilaton was not sufficient but maintained, at that time the $\mathrm{SpO}_{2}$, expired tidal volume and $\mathrm{ETCO}_{2}$ was between 90-95\%, 200-400 $\mathrm{ml}$ and 40-45 mmHg, respectively. But, a few seconds later, mask ventilation was more inadequate, leading to a decrease in the $\mathrm{SpO}_{2}$ up to $70 \%$ and cyanosis. Thus, LMA was immediately inserted for adequate ventilation, and the $\mathrm{SpO}_{2}$ were measured as $90 \%$, the patient's ventilation was smooth and $\mathrm{SpO}_{2}$ increased to $100 \%$, whereupon LMA-guided fiberoptic intuba- tion was successfully performed. Five minutes later, a pinkish foamy secretion was observed in the endotracheal tube, and pulmonary edema was shown by a portable chest radiograph; however, no other abnormality was observed (Fig. 1B). Arterial blood gas analysis (ABGA) at an inspired oxygen fraction of 0.5 showed the following: $\mathrm{pH} 7.3, \mathrm{PCO}_{2} 45 \mathrm{mmHg}, \mathrm{PO}_{2} 149 \mathrm{mmHg}$, and $\mathrm{BE}-2.5 \mathrm{mmol} / \mathrm{L}$. Judging that the pulmonary edema was not serious based on the ABGA and chest radiograph, we proceeded with the surgery as planned and intermittent the endotracheal tube suction for $30 \mathrm{~min}$ after initiation of the surgery, until the secretion stopped. The total amount of secretion was approximately $20 \mathrm{ml}$.

The surgery was completed successfully after $4.5 \mathrm{~h}$, and the patient was transferred to the post-anesthesia care unit. Upon improvement of pulmonary edema, verified using chest radiographs, the patient was transferred to the recovery room (Fig. 1C). The patient was moved to the general ward, where she did not show any signs of dyspnea or hypoxia. The chest radiograph taken on postoperative day 2 did not show any abnormality (Fig. 1D), and the patient was discharged from the hospital after 2 weeks.

The type of pulmonary edema frequently associated with airway management or general anesthesia is negative pressure pulmonary edema (NPPE), which is mainly caused by upper airway obstruction such as tracheal extubation, laryngospasm and airway tumor and high negative intrathoracic pressure of -50 to $-100 \mathrm{cmH}_{2} \mathrm{O}$ generated by respiratory effort, resulting in an abrupt increase in the venous return, leading to increased ventricular volume. This, in turn, increases the left ventricular end-diastolic pressure, leading to pulmonary edema [1]. In the

Corresponding author: Jong Hun Jun, M.D., Ph.D., Department of Anesthesiology and Pain Medicine, Hanyang University School of Medicine, 222, Wangsimni-ro, Seongdong-gu, Seoul 133-792, Korea. Tel: 82-2-2290-8680, Fax: 82-2-2299-8692, E-mail: jhjun@hanyang.ac.kr

(c) This is an open-access article distributed under the terms of the Creative Commons Attribution Non-Commercial License (http:// creativecommons.org/licenses/by-nc/3.0/), which permits unrestricted non-commercial use, distribution, and reproduction in any medium, provided the original work is properly cited. 

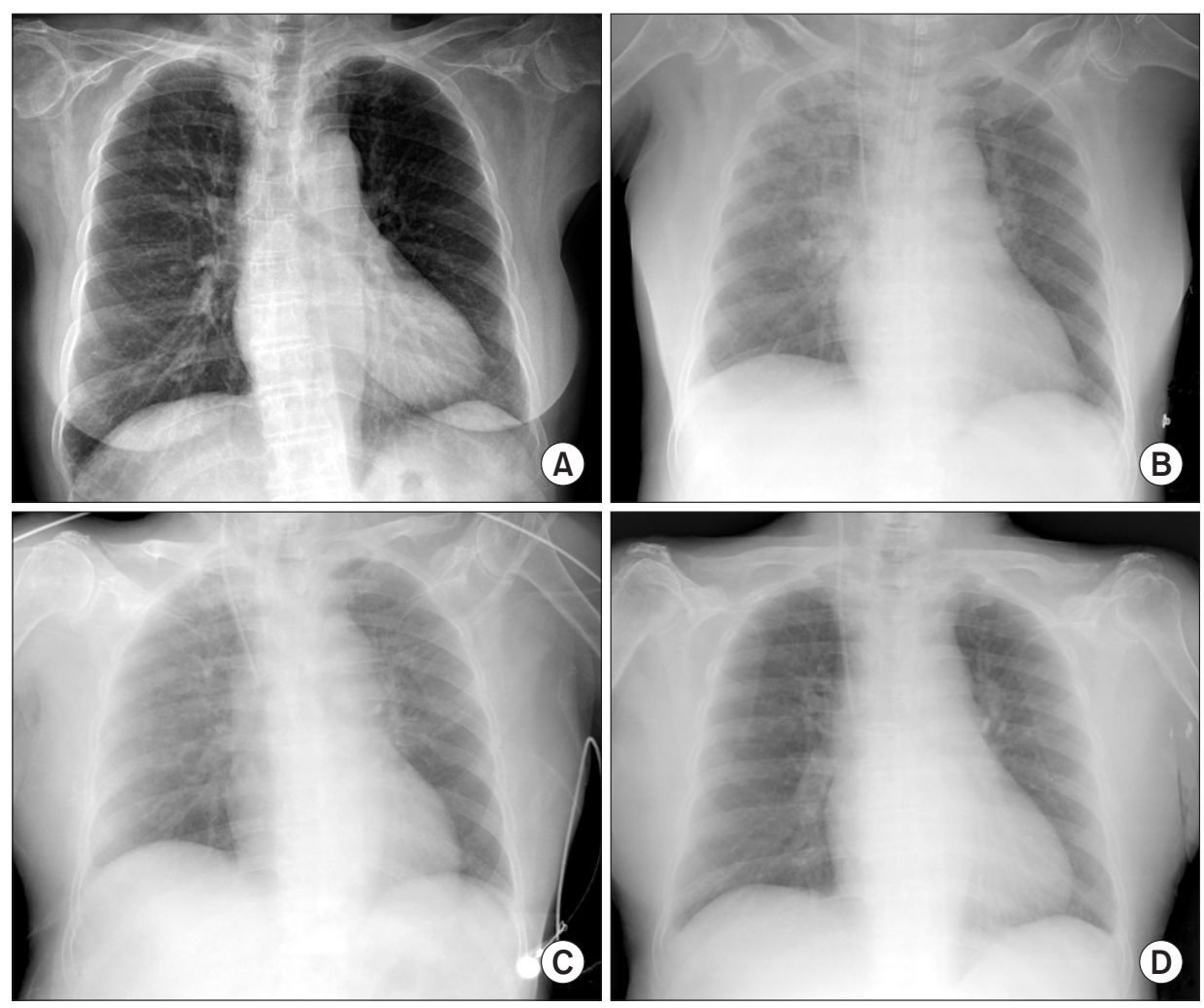

Fig. 1. (A) Chest radiograph prior to surgery showing suspicious bronchitis at the right lower lobe costophrenic angle area. (B) Chest radiograph after intubation showing pulmonary edema at both the upper lobes and no cardiomegaly. (C) Chest radiograph when the patient was at the post-anesthesia care unit showing improved pulmonary edema. (D) Chest radiograph after 2 days.

present case, however, no negative intrathoracic pressure was observed during the absence of spontaneous respiratory effort, which was induced by the injection of an intubation dose of a muscle relaxant during the induction of general anesthesia. The patient, thus, was exposed to hypoxia, which resulted from the 6 min insufficient ventilation that was associated with difficulties in intubation until adequate ventilation was achieved through LMA and pulmonary edema occurred immediately after this incident. Therefore, the cause of pulmonary edema in this case is believed to be hypoxia rather than NPPE.

High-altitude pulmonary edema (HAPE) is a type of pulmonary edema associated with hypoxia [2]. In hypoxia-induced HAPE, edema occurs in interstitial and alveolar spaces. HAPE is caused by poor ventilator response, increased sympathetic tone, exaggerated and uneven hypoxic pulmonary vasoconstriction, and inadequate production of hormonal mediators such as nitric oxide [3]. This case report involving pulmonary edema may have been induced, similar to that in the case of HAPE, by the increase in systemic vascular resistance and LV afterload, because of the increased sympathetic tone arising from the continuous stimulation of airway and hypoxic stimulation during intubation failure. Additionally, pulmonary artery constriction, which was triggered by alveolar hypoxia, resulted in an increase in pulmonary vascular resistance, leading to pulmonary hypertension [4]. This change may also indirectly result in an increase in the right ventricular pressure, leading to interventricular septal motion, causing a left ventricular diastolic dysfunction and contributed to the occurrence of pulmonary edema. In addition, a previous study using rats showed that hypoxia inhibits the resorption of fluid in the lungs through the inhibition of Na channels [2]. Thus, similar to that in HAPE, the above-mentioned multiple mechanisms may have caused acute pulmonary edema through hypoxia during anesthesia induction.

RA is primarily a systemic autoimmune disease involving the joints. However, reports have shown that the rate of lung involvement in RA patients is as high as 70\% [5]. Thus, RA patients are at risk to changes in the pulmonary conditions such as pulmonary fibrosis, lung nodules, bronchiolitis, tuberculosis, pleural effusions, and bronchiectasis. For these reasons, RA patients are believed to be at high risk for pulmonary injury.

In the present case, acute pulmonary edema improved without administering any specific treatment. The sympathetic tone stimulated by the airway and hypoxia was reduced by the application of inhalation anesthetics and opioids. Further, pulmonary edema improved due to prompt correction of hypoxia, thus, preventing further progression of the condition. 


\section{References}

1. Bisinotto FM, Cardoso Rde P, Abud TM. Acute pulmonary edema associated with obstruction of the airways. Case report. Rev Bras Anestesiol 2008; 58: 165-71.

2. Bärtsch P, Mairbäurl H, Maggiorini M, Swenson ER. Physiological aspects of high-altitude pulmonary edema. J Appl Physiol (1985) 2005; 98: 1101-10.

3. Birukov KG, Jacobson JR, Flores AA, Ye SQ, Birukova AA, Verin AD, et al. Magnitude-dependent regulation of pulmonary endothelial cell barrier function by cyclic stretch. Am J Physiol Lung Cell Mol Physiol 2003; 285: L785-97.

4. Aaronson PI, Robertson TP, Knock GA, Becker S, Lewis TH, Snetkov V, et al. Hypoxic pulmonary vasoconstriction: mechanisms and controversies. J Physiol 2006; 570: 53-8.

5. Harmon KR, Leatherman JW. Respiratory manifestations of connective tissue disease. Semin Respir Infect 1988; 3: 258-73. 